\title{
The Association Between Reflux Esophagitis and Psychosocial Stress
}

\author{
Eun Mi Song $\cdot$ Hye-Kyung Jung $\cdot$ Ji Min Jung
}

Received: 23 April 2012/Accepted: 18 August 2012/Published online: 22 September 2012

(C) The Author(s) 2012. This article is published with open access at Springerlink.com

\begin{abstract}
Background The prevalence of reflux esophagitis is increasing in Korea. Reflux esophagitis aggravates the stress and fatigue level of daily life, but less is known about the association with stress and fatigue, which could be bi-directional.

Aim To evaluate the impact of reflux esophagitis on stress and fatigue and to compare the stress level of people with reflux esophagitis with that of controls with peptic ulcer disease and healthy controls.

Methods Among a total of 9,033 subjects who underwent a comprehensive medical check-up including upper endoscopy, 6,834 subjects (75.7\%) were enrolled. Stress and fatigue scores were measured by a validated Korean version of the Brief Encounter Psychosocial Instrument and the Fatigue Severity Scale.

Results Among 6,834 subjects, $13.2 \%$ were in the highstress group, and reflux esophagitis was found in $6.0 \%$. After adjustment for confounders, reflux esophagitis was significantly associated with high stress (odds ratio 1.94, $95 \%$ confidence interval 1.25-3.02). Subjects with reflux esophagitis had significantly higher BEPSI-K scores compared with healthy controls $(p=0.027)$; and however, there was no significant difference in BEPSI-K scores between reflux esophagitis group and peptic ulcer disease controls. Fatigue severity scale was highly correlated with BEPSI-K $(p<0.001)$; however, there was no significant difference in fatigue severity scale level between the reflux esophagitis group and controls. The severity of reflux
\end{abstract}

E. M. Song · H.-K. Jung $(\bowtie) \cdot$ J. M. Jung Department of Internal Medicine, Ewha Womans University School of Medicine, 911-1 Mock-dong, Yangchun-ku, Seoul 158-710, Korea e-mail: junghk@ewha.ac.kr esophagitis was significantly correlated with BEPSI-K score $(p=0.008)$.

Conclusions Reflux esophagitis is significantly associated with psychosocial stress, and the severity of reflux esophagitis correlates with the degree of stress.

Keywords Gastroesophageal reflux - Stress - Fatigue . Peptic ulcer disease

\section{Introduction}

Gastro-esophageal reflux disease (GERD), one of the most prevalent gastrointestinal (GI) diseases in Western countries, is increasing in frequency in Asia [1]. The prevalence of symptom-based GERD in eastern Asia was reported as $6.2-7.1 \%$ in $2010[2,3]$, whereas it was reported to be $2.5-4.8 \%$ in $2005[1,4]$. Endoscopy-based studies showed that the prevalence of reflux esophagitis in eastern Asia was $3-4 \%$ before 2000 , whereas other studies reported rates of $6.6-15.5 \%$ from 2000 to 2005 and $4.3-15.7 \%$ after 2005 [5].

Epidemiologic changes in GERD in Asia seem to be correlated with economic or environmental effects, changing Helicobacter pylori epidemics, and nutritional changes. Body weights are on the rise, diets are becoming less healthy, and people are becoming increasingly sedentary, resulting in metabolic alterations that increase obesity. Additionally, modern society imposes demands that may increase chronic stress. Stress activates behavioral and physiological response patterns, and maladaptation to chronic stress might play a role in many diseases [6, 7].

Recently, many lines of evidence have suggested that stress, a measure of adverse psychosocial influences in adult life, may play an important role in GERD [8-10]. It 
has been shown that subjects who have been exposed to life stressors are more likely to complain of symptoms of GERD [8-10]. On the other hand, the presence of troublesome GERD symptoms affects one's daily life and can thereby induce stress [11]. Health-related quality of life (HRQoL) in patients with reflux esophagitis is impaired in physical, psychological, and social aspects, and other studies have found that patients with GERD report reduced work productivity [11, 12]. Reflux esophagitis aggravates the stress and fatigue levels of daily life, but less is known about the association between stress and fatigue, which could be bi-directional.

Health check-ups have recently become popular in Korea. Health check-ups are provided as a promotion by workplaces or are frequently performed at the patient's own expense, such as a cancer-screening program [13]. Most Korean hospitals are equipped with a Healthcare Center to do comprehensive health check-ups including endoscopy.

The aim of this study was to investigate the association between stress and reflux esophagitis on a large scale among people using a Healthcare Center, generally regarded as representative of the general population. First, we divided the study population according to stress level into two groups and evaluated factors associated with high stress level. Second, we compared stress levels among subjects who had reflux esophagitis, those with peptic ulcer disease (PUD), and healthy controls. PUD, which is well known to be a GI disease related to stress, was analyzed as a disease control. Additionally, we compared fatigue levels, which could reflect conditions resulting from chronic stress, among the reflux esophagitis group, PUD group, and controls.

\section{Materials and Methods}

Subjects

The study population consisted of 9,033 subjects who had medical check-ups at the Health Promotion Center of Ewha Womans University Mok-Dong Hospital. Among them, 6,903 subjects $(76.4 \%)$ who were over 18 years old and agreed to participate in this study were enrolled. For the baseline examination, physical examination, blood tests, and upper endoscopy were performed and questionnaires were administered (Fig. 1). The exclusion criteria were as follows: (1) subjects with prior major abdominal surgery $(n=18)$, except for appendectomy, cholecystectomy, or hysterectomy; (2) subjects with stomach cancer $(n=5)$; (3) subjects who had both reflux esophagitis and PUD $(n=46)$ (Fig. 1).

\section{Questionnaires}

Subjects were asked to complete a series of validated survey questionnaires to assess the severity of stress and fatigue.

\section{Stress Severity Scale (BEPSI-K)}

The modified Brief Encounter Psychosocial Instrument (BEPSI) [14, 15] was used in this study to measure the stress level. The BEPSI was developed as an instrument of stress measurement in a busy practice and is well correlated with other stress scales [15]. The Korean-language BEPSI (BEPSI-K) has been validated and is used broadly in health examinations [16]. The BEPSI-K consists of five items,
Fig. 1 Outline of the study subjects. The study population consisted of 6,834 subjects. We excluded the subjects who underwent previous abdominal surgery $(n=18)$, stomach cancer $(n=5)$, having reflux esophagitis and peptic ulcer disease

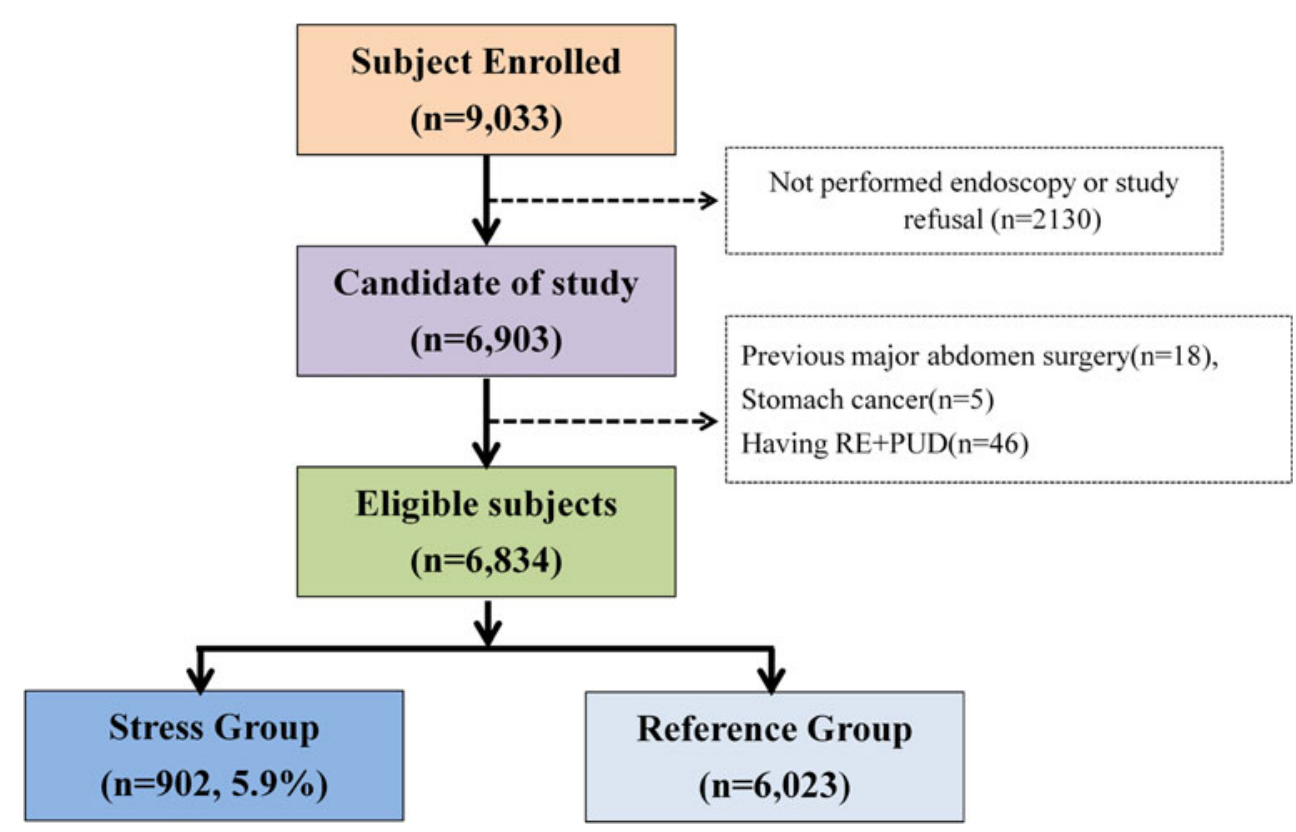


each of which is answered using a five-point Likert scale; the sum of the five items was divided by 5 for the final score. A subject with a higher score experiences more stress. The internal consistency of the scale was high (Cronbach's $\alpha$ : 0.84). A high stress level was defined as a BEPSI-K score of more than 2.4.

\section{Fatigue Severity Score (FSS)}

The Fatigue Severity Score (FSS) contains nine items developed to assess disabling fatigue [17]; the Korean version was validated in patients with multiple sclerosis, systemic lupus erythematosus, and chronic hepatitis C [18]. Item responses were measured on seven-point Likert-type scales ranging from strongly disagree to strongly agree. The nine items were combined into a total score; a lower total score indicates less effect of fatigue on everyday life. If responses to more than five items were missing, the FSS total score was considered missing. The internal consistency (Cronbach's $\alpha$ : 0.93) and test-retest reliability of the FSS were proven to be excellent in previous studies [19]. There is no general consent about what constitutes high FSS level. Therefore, we defined the high-fatigue group as the uppermost quartile according to the FSS.

\section{Demographics and Other Variables}

Demographic data and predictors for reflux esophagitis and psychosocial stress levels were as follows:

1. Demographics: gender, age

2. Specific factors related to reflux esophagitis and stress levels: (1) the presence of disease: hypertension (HTN), diabetes (DM); (2) alcohol consumption (drinking alcohol more than 2-3 times a week); (3) smoking (current smoker vs. non-smoker); (4) intake of coffee; (5) regular exercise; (6) obesity (body mass index $\quad\left(B M I \geq 25 \mathrm{~kg} / \mathrm{m}^{2}\right) ; \quad$ (7) serious obesity (BMI $\geq 30 \mathrm{~kg} / \mathrm{m}^{2}$ ); (8) education level (less than college graduate was categorized as lower education); (9) marital status (married vs. other); (10) income per month (earning less than US $\$ 4000$ ( $\$ 1 \approx 1,000$ won) was categorized as low income).

\section{Endoscopic Examinations}

All study subjects underwent upper-GI endoscopic examinations, which were performed by two well-trained gastroenterologists, each of whom had at least 5 years of endoscopy experience. The presence and severity of reflux esophagitis were classified using the Los Angeles (LA) classification as grade A-D (LA-A to D) and were based on the longest length of a mucosal break and the confluence of erosions [20]. Minimal change in reflux esophagitis was excluded because of poor reliability. PUD was defined as having an active or healing-stage gastric and/or duodenal ulcer; however, the scar stage of PUD was excluded.

\section{Statistical Analysis}

The $\chi^{2}$ test was used for categorical comparisons, and oneway analysis of variance with post hoc group differences corrected for multiple comparisons (Bonferroni correction) was used for comparison of continuous variables between the stress group and the reference group.

We performed correlation analyses using the Pearson product-moment correlation coefficient between BEPSI-K scores and FSS scores and the variables of age, income, and reflux grade. Student's $t$ tests were used to compare BEPSI-K scores and FSS scores between different groups based on gender, underlying disease (reflux esophagitis, PUD, DM, HTN), health-related behaviors (smoking habits, alcohol use, coffee, regular exercise, obesity), and socioeconomic factors (marital status, education). No corrections for multiple comparisons were made at this time. The results from these analyses were primarily used to develop a model explaining factors independently associated with BEPSI-K scores and FSS by entering factors associated with BEPSI-K scores and FSS at $p<0.05$ into a standard multiple linear regression analysis. Unless otherwise stated, significance was accepted at the $5 \%$ level, and the data are presented as mean \pm standard deviation.

\section{Ethics Statement}

This study was approved by the institutional review board of Ewha Womans University Mok-dong Hospital.

\section{Results}

Baseline Characteristics of the Study Subjects

A total of 6,834 subjects were included in this study. Women accounted for $45 \%$ of subjects, and the mean age of all subjects $( \pm \mathrm{SD})$ was $43.9( \pm 9.8)$ years. Reflux esophagitis was found in $407(6.0 \%)$ and active to healingstage PUD was found in 404 subjects (gastric ulcer, $n=291$; duodenal ulcer, $n=171$; gastric and duodenal ulcer, $n=58)$.

The study population was classified into two groups according to BEPSI-K scores: the stress group and the reference group. The stress group was defined as those having a BEPSI-K score $>2.4$. A total of 902 subjects were included in the stress group, with a mean BEPSI-K score of $2.92 \pm 0.61$ (Table 1). Compared with the reference 
Table 1 Baseline clinical characteristics in the stress group and reference group

\begin{tabular}{lllr}
\hline & $\begin{array}{l}\text { Stress group } \\
(n=902)(\%)\end{array}$ & $\begin{array}{l}\text { Reference group } \\
(n=5,936)(\%)\end{array}$ & $p$ value \\
\hline Female gender, $N(\%)$ & $420(46.6 \%)$ & $2,519(42.4 \%)$ & 0.011 \\
Age (years) & $42.3 \pm 10.5$ & $44.1 \pm 9.7$ & $<0.001$ \\
Hypertension & $318(35.7 \%)$ & $2,100(36.0 \%)$ & 0.881 \\
Diabetes mellitus & $37(4.2 \%)$ & $263(4.5 \%)$ & 0.727 \\
Current smoker & $302(35.7 \%)$ & $1,539(27.6 \%)$ & $<0.001$ \\
Exercise & $232(64.8 \%)$ & $1,689(75.4 \%)$ & 0.270 \\
Coffee & $305(84.3 \%)$ & $1,996(90.2 \%)$ & $<0.001$ \\
Alcohol & $500(76.1 \%)$ & $4,742(76.7 \%)$ & 0.734 \\
Married status & $717(79.5 \%)$ & $4,687(79.0 \%)$ & 0.930 \\
BMI $\geq 30 \mathrm{~kg} / \mathrm{m}^{2}$ & $40(4.5 \%)$ & $159(2.7 \%)$ & 0.006 \\
BMI $\geq 25 \mathrm{~kg} / \mathrm{m}^{2}$ & $218(33.7 \%)$ & $1,902(31.3 \%)$ & 0.212 \\
Low education & $78(8.8 \%)$ & $367(6.3 \%)$ & 0.007 \\
Low income & $245(31.4 \%)$ & $1,312(24.2 \%)$ & $<0.001$ \\
Reflux esophagitis & $74(8.2 \%)$ & $333(5.6 \%)$ & 0.003 \\
Peptic ulcer disease & $64(7.1 \%)$ & $340(5.7 \%)$ & 0.111 \\
FSS level & $4.26 \pm 1.30$ & $2.98 \pm 1.27$ & $<0.001$ \\
BEPSI-K level & $2.92 \pm 0.61$ & $1.49 \pm 0.39$ & $<0.001$ \\
\hline Dan & &
\end{tabular}

Data are presented as mean \pm standard deviation. BMI body mass index, FSS fatigue severity score

group, women were more common, and the mean age was younger in the stress group. The proportion of coffee drinkers was relatively smaller in the stress group. Subjects who were current smokers and those with low education and low income were more common in the stress group than the reference group. Subjects with reflux esophagitis were more prevalent in the stress group compared with the reference group ( 8.2 vs. $5.6 \%, p=0.003$ ). The prevalence of PUD in the stress group was higher than that in the reference group; however, the difference did not reach statistical significance (7.1 vs. $5.7 \% ; p=0.111)$. Seriously obese people (BMI $\geq 30 \mathrm{~kg} / \mathrm{m}^{2}$ ) were more common in the high-stress group (4.5 vs. $2.7 \% ; p=0.006$ ).

Univariate and Multivariate Analysis of Predictors for the Stress Group

In univariate analysis, female gender, current smoking, serious obesity (BMI $\geq 30 \mathrm{~kg} / \mathrm{m}^{2}$ ), low income, low education status, and high fatigue level all had significantly increased odds ratios in the high-stress group. However, aging and coffee drinking were inversely associated with high stress. Hypertension, diabetes mellitus, PUD, alcohol use, regular exercise, marital status, and obesity (BMI $\geq 25 \mathrm{~kg} / \mathrm{m}^{2}$ ) were not significantly related with high stress (Table 2).

In multivariate analysis, reflux esophagitis was a significant independent predictor for high stress (OR, 1.94; $95 \%$ CI, 1.25-3.02; $p<0.001$ ). Current smoking (OR,
$1.73 ; 95 \%$ CI, 126-2.36; $p<0.001)$ and low income (OR, $1.34 ; 95 \% \mathrm{CI}, 1.02-1.79 ; p<0.33$ ) were also significant predictors of psychosocial stress. High fatigue level was significantly associated with high stress level (OR, 3.79; CI $2.93-4.90 ; p<0.001)$. However, coffee consumption had a protective effect against stress (OR, 0.64; $95 \% \mathrm{CI}$, $0.43-0.94 ; p=0.021)$.

Comparison of Stress Levels in the Reflux Esophagitis, Peptic Ulcer Disease, and Control Groups

The stress level of the reflux esophagitis group was compared with that of the PUD group and controls. Subjects with reflux esophagitis had significantly higher BEPSI-K scores compared with controls $(1.75 \pm 0.67$ vs. $1.68 \pm 0.64$, $p=0.027)$. BEPSI-K scores were also higher in the reflux esophagitis group than in the PUD group, but the difference did not reach statistical significance $(1.75 \pm 0.67$ vs. $1.70 \pm 0.67, p=0.290)$. There was no significant difference in BEPSI-K score between the PUD group and controls $(1.70 \pm 0.66$ vs. $1.68 \pm 0.64, p=0.429)$ (Fig. 2).

The mean BEPSI-K score in LA-C reflux esophagitis patients was $2.05 \pm 0.96$, that in LA-B was $1.78 \pm 0.70$, and that in LA-A was $1.74 \pm 0.66$. The severity of reflux esophagitis was positively correlated with BEPSI-K score in correlation analysis $(r=0.032, p=0.008)$ (Fig. 3). The proportion of subjects with high BEPSI-K scores (BEPSI$K \geq 2.4$ ) was greater in the reflux esophagitis group compared with the control group (13.5 vs. $9.4 \%)(p<0.001)$.

Comparison of Fatigue Score in the Reflux Esophagitis, Peptic Ulcer Disease, and Control Groups

BEPSI-K scores were positively correlated with FSS $(p<0.001)$, but there was no significant difference in FSS level between the reflux esophagitis and control groups $(3.12 \pm 1.33$ vs. $3.17 \pm 1.34, p=0.631)$. Also, there was no significant difference in FSS level between the PUD and control groups $(3.02 \pm 1.34$ vs. $3.17 \pm 1.34, p=0.569)$.

\section{Discussion}

The key finding in the present study was an association of psychosocial stress and reflux esophagitis, which is highly prevalent in Korea. In subjects with reflux esophagitis, the OR was 1.94 for high stress after adjusting for several confounders, and the mean stress score in the reflux esophagitis group was higher than that in PUD or controls. Furthermore, the stress score showed a significantly increasing trend with increasingly severe grade of reflux esophagitis. 
Table 2 Significant predictors of high stress level by multiple regression analysis

\begin{tabular}{|c|c|c|c|c|c|c|}
\hline & \multicolumn{3}{|c|}{ Univariate analysis } & \multicolumn{3}{|c|}{ Multivariate analysis } \\
\hline & OR & $95 \% \mathrm{CI}$ & $p$ value & OR & $95 \% \mathrm{CI}$ & $p$ value \\
\hline Female gender & 1.16 & $1.02-1.31$ & 0.011 & 1.19 & $0.88-1.61$ & 0.131 \\
\hline Age $\geq 40$ years & 0.72 & $0.62-0.83$ & $<0.001$ & 1.19 & $0.91-1.56$ & 0.216 \\
\hline Hypertension & 0.99 & $0.85-1.14$ & 0.881 & & & \\
\hline Diabetes mellitus & 0.92 & $0.65-1.31$ & 0.727 & & & \\
\hline Current smoker & 1.43 & $1.23-1.67$ & $<0.001$ & 1.73 & $1.26-2.36$ & $<0.001$ \\
\hline Exercise & 0.60 & $0.97-1.34$ & 0.270 & & & \\
\hline Coffee & 0.58 & $0.42-0.80$ & $<0.001$ & 0.64 & $0.43-0.94$ & 0.021 \\
\hline Alcohol & 0.96 & $0.80-1.15$ & 0.734 & & & \\
\hline Married state & 1.01 & $0.85-1.20$ & 0.930 & & & \\
\hline $\mathrm{BMI} \geq 25 \mathrm{~kg} / \mathrm{m}^{2}$ & 1.10 & $0.95-1.29$ & 0.212 & & & \\
\hline $\mathrm{BMI} \geq 30 \mathrm{~kg} / \mathrm{m}^{2}$ & 1.54 & $1.16-2.04$ & 0.006 & 1.41 & $0.76-2.59$ & 0.242 \\
\hline Low income & 1.37 & $1.19-1.51$ & $<0.001$ & 1.34 & $1.01-1.77$ & 0.041 \\
\hline Low education & 1.36 & $1.09-1.68$ & 0.007 & 0.69 & $0.34-1.42$ & 0.326 \\
\hline Reflux esophagitis & 1.50 & $1.16-1.95$ & 0.003 & 1.94 & $1.25-3.02$ & $<0.001$ \\
\hline PUD & 1.26 & $0.95-1.66$ & 0.111 & & & \\
\hline High fatigue level & 4.73 & $4.08-5.49$ & $<0.001$ & 3.79 & $2.93-4.90$ & $<0.001$ \\
\hline
\end{tabular}

$B M I$ body mass index, $P U D$ peptic ulcer disease

Fig. 2 Comparison of stress levels or fatigue severity score among the reflux esophagitis group, peptic ulcer disease (PUD) group, and controls group. *, comparison between reflux esophagitis versus controls; **, comparison between reflux esophagitis versus peptic ulcer disease. BEPSI-K score was significantly higher in reflux esophagitis group
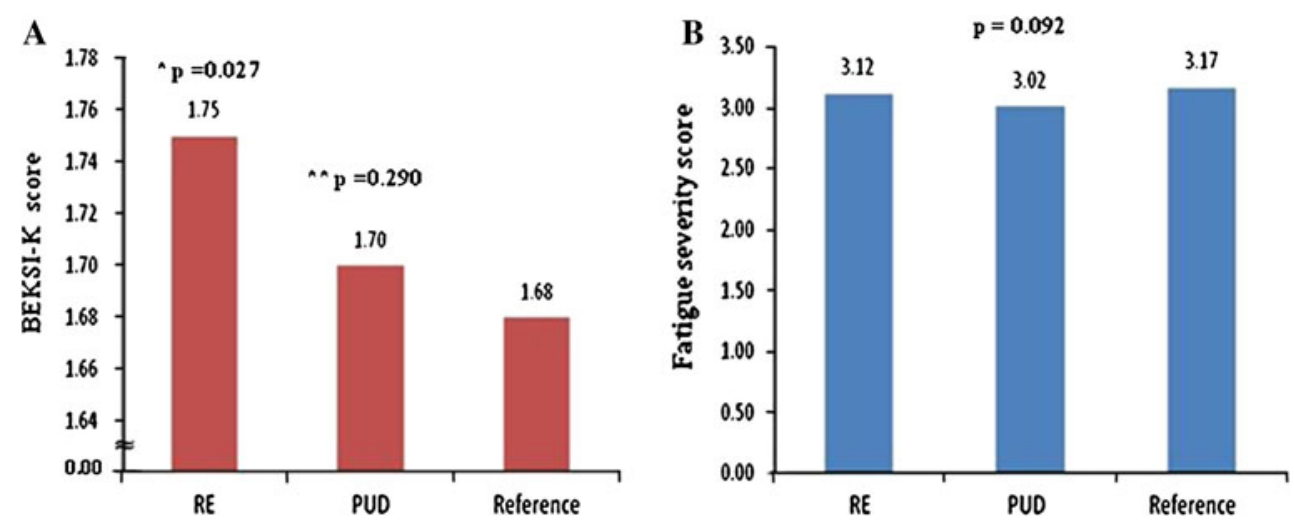

The medical literature on humans and animals strongly indicates that stress has adverse effects on the organism. In populations of patients with coronary heart disease, prospective studies showed that depression, anxiety, and stress may cause coronary heart disease and affect survival [6]. Psychosocial factors including stress also alter the function of the GI tract and GI symptom perception in patients with GERD. The relationship of stress to GI function is viewed as a direct consequence of bidirectional modulation by the central nervous system, including motor responses, pain modulation, and even immune function [21].

Previous studies have shown that stress can produce altered GI motility and symptoms. In a Gallup Poll, $64 \%$ of individuals with heartburn, the major symptom of GERD, reported that stress increased their symptoms [22]. Stress can exacerbate heartburn symptoms in GERD

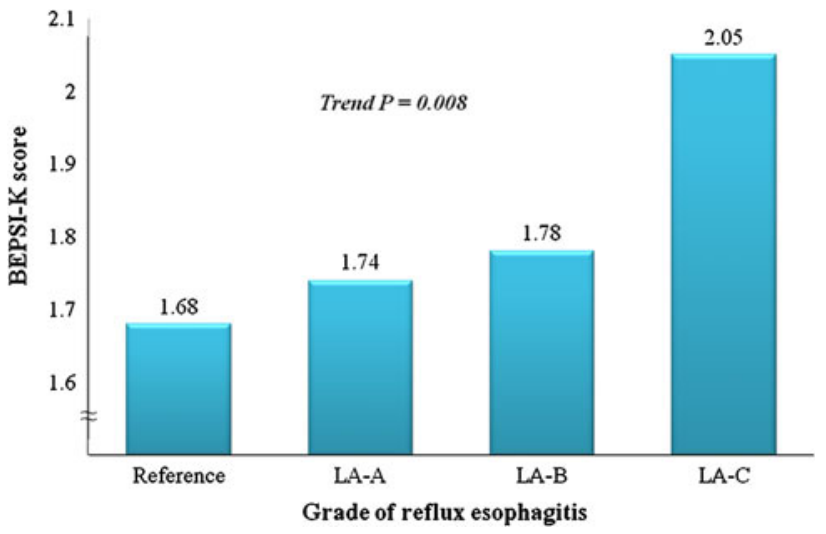

Fig. 3 Comparison of BEPSI-K according to severity of reflux esophagitis. The severity of reflux esophagitis was positively correlated with BEPSI-K score $(p$ value for trend $=0.008$ ) 
patients by enhancing perceptual response to intra-esophageal acid exposure [21,23]. Also, many other studies have reported increased frequency of GERD symptoms under specific stressful conditions [24]. In one study, stress did not influence objective measurements of acid reflux; however, chronic reflux patients who were chronically anxious and exposed to prolonged stressful stimuli may be more likely to perceive low-intensity esophageal stimuli as painful reflux symptoms [8].

In a previous study, higher levels of anxiety induced an overall tendency toward nonpropulsive activity of the esophagus [9]. Acute stressors increased resting loweresophageal sphincter pressure and impaired sphincter relaxation, thus delaying acid clearance from the esophagus [25]. In a study that assessed gastric acid output in relation to personality traits, it was found that subjects who were considered to have a higher level of impulsivity and expressed emotions more freely were more likely to react with a marked increase in gastric acid output when stimulated by stress [10]. These results suggest that stress could actually induce objective reflux of gastric contents and eventually result in reflux esophagitis regardless of symptom presence. Furthermore, stress is believed to induce reflux esophagitis by increasing esophageal mucosal permeability. In the experimental rat model, acute stress increases submucosal mast cell and acid pepsin mucosal permeability, and electron microscopy showed dilated intercellular spaces in mucosa from stressed rats [26].

Stress may affect health-related behaviors such as smoking, diet, alcohol consumption, or physical activity, which in turn may influence the risk of reflux esophagitis. Alcohol drinking and cigarette smoking exacerbate reflux by diminishing lower esophageal sphincter pressure [27, 28]. These behaviors could result in increase of reflux in stress condition. Also, exposure to chronic psychological stressors causes some individuals to consume food in excess of requirements, and then this may culminate in obesity [29]. Obesity has been established as a risk factor for GERD [30]. In the present study, confounding variables such as smoking, high obesity (BMI $\geq 30 \mathrm{~kg} / \mathrm{m}^{2}$ ) were significantly related to stress. However, multivariate analysis showed that having reflux esophagitis was an independent predictor for stress after adjusting for these factors.

On the other hand, chronic reflux symptoms could be a very bothersome experience in daily living, and the associated sleep disturbance or decreased work productivity can be another important source of stress [31]. The higher level of stress in the reflux esophagitis group may represent the impact of troublesome reflux symptoms on HRQoL in individuals. Wang et al. [32] demonstrated that GERD was associated with significantly impaired HRQoL and significant daytime sleepiness in Chinese individuals.
The relationship between stress and reflux esophagitis is distinctive. The positive correlation observed between reflux esophagitis and stress was not demonstrated in the PUD group. PUD is another GI disease that has been associated with stress, although the role of psychosocial factors in the development of ulcers remains incompletely understood [33]. The effect of stress on personal traits and high acid secretion may play an important role in the pathogenesis of PUD. However, the role of infection with H. pylori and the use of non-steroidal anti-inflammatory drugs might be more important in the pathophysiology of PUD; in the present study, these factors were not evaluated.

We also compared fatigue levels in the reflux esophagitis, PUD, and control groups. Fatigue score was positively correlated with BEPSI-K score; however, fatigue levels were not significantly different among the three groups. In one study, vital exhaustion, a cardinal symptom of fatigue, was an independent predictor of aggravations of heartburn [34]. Fatigue, which may in part result from sustained stress, may represent a psychophysiological symptom complex and has a close relationship with chronic conditions such as multiple sclerosis and chronic liver disease. Fatigue may result from cumulative stressful events. However, a correlation of fatigue with active reflux esophagitis or PUD was not found in the present study.

The present study has several strengths. First, this study enrolled a large sample of 6,834 ethnically homogenous participants with a high response rate $(75.7 \%)$. Two-thirds of the study population from the Healthcare Center in the present study were offered a routine health screening check-up as a reward, and only $1 / 3$ paid their own expenses. The study population might be a representative subgroup of the general population. Second, the present study was conducted with high-quality methodology. We used structured translated questionnaires with cross-cultural validation $[16,18]$. Reflux esophagitis was evaluated with upper endoscopy, the gold standard for the diagnosis of esophagitis, by experienced specialists in endoscopy. Also, all subjects underwent endoscopy. A comprehensive medical check-up in Korea usually includes upper endoscopy because of the high incidence of gastric cancer and relatively cheap cost.

We also acknowledge some limitations. First, we used a self-report system to collect our data. Recall and responder bias may affect the interpretation of results, although we have no reason to suspect that this bias was different among the case and control groups, and we used well-validated questionnaires. Another limitation is that we enrolled individuals who underwent medical check-ups. There might be selection bias; a previous study in Korea demonstrated that the health-screening population had higher income and educational status compared with the general Korean population. However, other factors such as BMI, smoking, 
alcohol habit, or co-morbidities were comparable to the background population [13]. Furthermore, the participation rate in the present study was acceptable, and the study scale was huge.

In summary, we found the perceived stress level measured by a standardized, well-established instrument to be higher in individuals with reflux esophagitis. The present study clearly suggests that psychosocial stress might have a significant relationship with reflux esophagitis and may have a potential role in the symptom presentation and natural history. Clinicians need to be sensitive to the close association between stress and reflux esophagitis and the strong possibility that patients with refractory GERD may have comorbid psychosocial health problems.

\section{Conflict of interest None.}

Open Access This article is distributed under the terms of the Creative Commons Attribution Noncommercial License which permits any noncommercial use, distribution, and reproduction in any medium, provided the original author(s) and the source are credited.

\section{References}

1. Fock KM, Talley NJ, Fass R, et al. Asia-Pacific consensus on the management of gastroesophageal reflux disease: update. $J$ Gastroenterol Hepatol. 2008;23:8-22.

2. Wang R, Ma XQ, Cao Y, et al. Burden of gastroesophageal reflux disease in Shanghai, China. Dig Liver Dis. 2009;41:110-115.

3. Yang SY, Lee OY, Bak YT, et al. Prevalence of gastroesophageal reflux disease symptoms and uninvestigated dyspepsia in Korea: a population-based study. Dig Dis Sci. 2008;53:188-193.

4. Goh KL, Chang SC, Fock KM, Ke M, Park HJ, Lam SK. Gastrooesophageal reflux disease in Asia. $J$ Gastroenterol Hepatol. 2000;15:230-238.

5. Jung HK. Epidemiology of gastroesophageal reflux disease in Asia: a systematic review. J Neurogastroenterol Motil. 2011;17:14-27.

6. Hemingway H, Marmot M. Evidence based cardiology: psychosocial factors in the aetiology and prognosis of coronary heart disease. Systematic review of prospective cohort studies. BMJ. 1999;318:1460-1467.

7. Hjemdahl P. Stress and the metabolic syndrome: an interesting but enigmatic association. Circulation. 2002;106:2634-2636.

8. Bradley LA, Richter JE, Pulliam TJ, et al. The relationship between stress and symptoms of gastroesophageal reflux: the influence of psychological factors. Am J Gastroenterol. 1993;88:11-19.

9. Rubin J, Nagler R, Spiro HM, Pilot ML. Measuring the effect of emotions on esophageal motility. Psychosom Med. 1962;24: $170-176$.

10. Holtmann G, Kriebel R, Singer MV. Mental stress and gastric acid secretion. Do personality traits influence the response? Dig Dis Sci. 1990;35:998-1007.

11. Kulig M, Leodolter A, Vieth M, et al. Quality of life in relation to symptoms in patients with gastro-oesophageal reflux disease- an analysis based on the ProGERD initiative. Aliment Pharmacol Ther. 2003;18:767-776.

12. Henke CJ, Levin TR, Henning JM, Potter LP. Work loss costs due to peptic ulcer disease and gastroesophageal reflux disease in a health maintenance organization. Am J Gastroenterol. 2000; 95:788-792.
13. Kim N, Lee SW, Cho SI, et al. The prevalence of and risk factors for erosive oesophagitis and non-erosive reflux disease: a nationwide multicentre prospective study in Korea. Aliment Pharmacol Ther. 2008;15:173-185.

14. Frank SH, Zyzanski SJ. Stress in the clinical setting: the Brief Encounter Psychosocial Instrument. J Fam Pract. 1988;26: 533-539.

15. Yim JH, Bae JM, Choi SS. The validity of modified Korean translated BEPSI as instrument of stress measurement in outpatient clinic. J Korean Acad Fam Med. 1996;17:42-49.

16. Bae JM, Jeong EK, Yoo TW, Huh BY. A quick measurement for stress in outpatient clinic setting. J Korean Acad Fam Med. 1992;13:809-820.

17. Krupp LB, LaRocca NG, Muir-Nash J, Steinberg AD. The fatigue severity scale. Application to patients with multiple sclerosis and systemic lupus erythematosus. Arch Neurol. 1989;46:1121-1123.

18. Chung KI, Song CH. Clinical usefulness of fatigue severity scale for patients with fatigue, and anxiety or depression. Korean J Psychosom Med. 2001;9:164-173.

19. Valko PO, Bassetti CL, Bloch KE, Held U, Baumann CR. Validation of the fatigue severity scale in a Swiss cohort. Sleep. 2008;31:1601-1607.

20. Lundell LR, Dent J, Bennett JR, et al. Endoscopic assessment of oesophagitis: clinical and functional correlates and further validation of the Los Angeles classification. Gut. 1999;45:172-180.

21. Levy RL, Olden KW, Naliboff BD, et al. Psychosocial aspects of the functional gastrointestinal disorders. Gastroenterology. 2006;130:1447-1458.

22. Corneille MG, Gallup TM, Villa $\mathrm{C}$, et al. Pediatric vascular injuries: acute management and early outcomes. $J$ Trauma. 2011;70:823-828.

23. Wright CE, Ebrecht M, Mitchell R, Anggiansah A, Weinman J. The effect of psychological stress on symptom severity and perception in patients with gastro-oesophageal reflux. $J$ Psychosom Res. 2005;59:415-424.

24. Fass R, Naliboff BD, Fass SS, et al. The effect of auditory stress on perception of intraesophageal acid in patients with gastroesophageal reflux disease. Gastroenterology. 2008;134:696-705.

25. Richter JE, Bradley LC. Psychophysiological interactions in esophageal diseases. Semin Gastrointest Dis. 1996;7:169-184.

26. Peterson WL. The role of acid in upper gastrointestinal haemorrhage due to ulcer and stress-related mucosal damage. Aliment Pharmacol Ther. 1995;9:43-46.

27. Kahrilas PJ, Gupta RR. Mechanisms of acid reflux associated with cigarette smoking. Gut. 1990;31:4-10.

28. Vitale GC, Cheadle WG, Patel B, et al. The effect of alcohol on nocturnal gastro-esophageal reflux. JAMA. 1987;258:2077-2079.

29. Torres SJ, Nowsnon CA. Relationship between stress, eating behavior, and obesity. Nutrition. 2007;23:887-894.

30. Dore MP, Maragkoudakis E, Fraley K, et al. Diet, lifestyle and gender in gastro-esophageal reflux disease. Dig Dis Sci. 2008; 53:2027-2032.

31. Choi JY, Jung HK, Roh SH, et al. Sleep disorder associated with gastroesophageal reflux disease. Korean J Med. 2011;81: 602-610.

32. Wang R, Zou D, Ma X, et al. Impact of gastroesophageal reflux disease on daily life: the Systematic Investigation of Gastrointestinal Diseases in China (SILC) epidemiological study. Health Qual Life Outcomes. 2010;8:128.

33. Jones MP. The role of psychosocial factors in peptic ulcer disease: beyond Helicobacter pylori and NSAIDs. J Psychosom Res. 2006;60:407-412.

34. Naliboff BD, Mayer M, Fass R, et al. The effect of life stress on symptoms of heartburn. Psychosom Med. 2004;66:426434. 